Main Outcome Measurements Brain activity was measured through electroencephalography during both balance tests and computed by means of spectral power analysis. The predefined balance task was the Y-balance test (YBT), while the reactive balance test (RBT) was selected as the neurocognitive balance test. Results For the YBT, $\theta$-power of the prefrontal cortex significantly increased due to MF. For the RBT, brain activity remained unchanged. RBT accuracy worsened $(\mathrm{p}<0.05)$ following MF, while YBT performance did not significantly change.

Conclusions The MF intervention induced unfavourable brain activity changes during the YBT, but did not affect YBT performance. However, no changes in brain electrophysiological functioning were observed during RBT execution while a decrease in RBT accuracy occurred. These results suggest that sustained attention tasks might affect different central mechanisms depending on the type of balance task. Further research is needed in order to elucidate the role of the brain and MF in balance performance and injury occurrence.

\section{PSYCHOLOGICAL FACTORS FOR AN INJURY FREE ATHLETIC CAREER}

1,2Ulrika Tranaeus, 'Linn Hallgren, ${ }^{1}$ Hanna Jörlund. 'The Swedish School of Sport and Health Sciences, GIH, Stockholm, Sweden; ${ }^{2}$ Karolinska Institutet, Stockholm, Sweden

\subsection{6/bjsports-2021-IOC. 130}

Background Many athletes remain uninjured during their careers. Little is known about if and which psychological factors that may contribute to protect from athletic injuries.

Objective Do uninjured athletes use buffering coping skills and/ or other protecting psychological factors to remain injury free?

Design Qualitative interview study.

Setting Face to face interviews, using a semi-structured interview guide with open questions, with Swedish elite athletes.

Patients (or Participants) Six athletes (four males and two females), 23-25 years old, competing at elite level in team sports or running without severe time-loss injuries during their careers volunteered to participate. The definition of uninjured was less than a total of four weeks absence from training due to injury or pain. Time for competing varied from five years to nineteen years.

Main Outcome Measurements The inductive thematic content analysis was aiming to identity common psychological factors in the narratives.

Results The analysis showed six themes that were suggested to influence the athletes to perform and compete without sustaining severe injuries. The themes with examples of underlying categories were 1) structure during the daily life (time management, logistic), 2) all-round training (late specialisation), 3) knowledge (education), 4) coping strategies (acceptance, goal oriented), 5) recovery strategies (body awareness, food and sleep habits) and 6) social support (support from coaches and parents).

Conclusions Adaptive coping strategies and identified psychological factors are suggested to have possible impact on athletes' potential to stay uninjured. This study can play a role of generating hypotheses for testing in future studies, until then athletes and coaches are encouraged to bear these findings in mind in order to prevent injuries and support athletes.

\section{1} THE EFFECT OF FATIGUE ON TRUNK AND PELVIC JUMPLANDING BIOMECHANICS: A SYSTEMATIC REVIEW

${ }^{1,2}$ Stefan Vermeulen, ${ }^{1,2}$ Camilla De Bleecker, ${ }^{1}$ Cedric De Blaiser, ${ }^{3}$ Özge Onursal Kilinc, ${ }^{1}$ Tine Willems, ${ }^{2}$ Jos Vanrenterghem, ${ }^{1}$ Philip Roosen, ${ }^{1}$ Roel De Ridder. 'University of Ghent, Department of Rehabilition Sciences, Ghent, Belgium; ${ }^{2} \mathrm{KU}$ Leuven, Leuven, Belgium; ${ }^{3}$ Hacettepe University, Ankara, Turkey

\subsection{6/bjsports-2021-IOC.131}

Background Physical active induced fatigue may result altered trunk and pelvic biomechanics during athletic tasks.

Objective To summarize and determine the effect of physical fatigue on trunk and pelvic biomechanics during jump-landings in healthy, physically active populations.

Design Systematic review of literature.

Setting Electronic databases PubMed, Web of Science, Embase, CINAHL, and SPORTDiscus were consulted up to and including April 2021.

Patients (or Participants) Cross-sectional studies including healthy, physically active populations.

Interventions (or Assessment of Risk Factors) Two reviewers independently screened for relevant studies. Methodological quality was assessed by a modified Downs and Black checklist. Main Outcome Measurements Studies investigating the effect of fatigue on trunk and pelvic kinematics and/or muscular activity during double- and single-legged jump-landings and jump-landing related cutting tasks in healthy, physically active populations.

Results Eighteen studies investigating trunk and pelvic landing biomechanics before and after fatigue met the inclusion criteria. Methodological quality was moderate to high among the included studies. The results of the systematic review show a wide variability of trunk and pelvic landing strategies after fatigue. Based on the kinematic results, there is evidence for more trunk flexion during standardized jump-landings after fatigue $(p=0,001-0,007)$. Regarding muscular activity, there is no evidence of fatigue-related alterations in lumbo-pelvic muscular activations during landing.

Conclusions The results of this systematic review provide evidence for adaptive trunk and pelvic biomechanical strategies during landing after fatigue to potentially unload fatigued lower extremity musculoskeletal structures.

\section{THE FATIGUE-INJURY HYPOTHESIS: WHAT IS THE EFFECT OF ACUTE PERIPHERAL FATIGUE ON FUNCTIONAL AND NEUROCOGNITIVE PERFORMANCE TESTS?}

${ }^{1}$ Jo Verschueren, 'Bruno Tassignon, ${ }^{2}$ Evert Verhagen, ${ }^{1,3}$ Romain Meeusen. ${ }^{1}$ Human Physiology Research Group, Faculty of Physical Education and Physiotherapy, Vrije Universiteit Brussel, Brussels, Belgium; ${ }^{2}$ Amsterdam Collaboration on Health and Safety in Sports, Department of Public and Occupational Health, Amsterdam Movement Sciences, Amsterdam UMC, Vrije Universiteit Amsterdam, Amsterdam, Netherlands; ${ }^{3}$ Strategic Research Program Exercise and the Brain in Health and Disease: the added value of HumanCentered Robotics, Vrije Universiteit Brussel, Brussels, Belgium

\subsection{6/bjsports-2021-IOC.132}

Background Physical fatigue is known to decrease an athlete's functional test performance (FPT), but less is known about the impact on the injury risk profile. Furthermore, adaptability and neurocognitive performance tests have been put forward as relevant concepts within injury prevention, but to date it is not known if acute peripheral fatigue affects functional and neurocognitive performance tests. 